\title{
Pulse-by-pulse near 800-nm band power stabilization using all-optical limiter based on self-phase modulation
}

\author{
Takuya Murakawa, and Tsuyoshi Konishi \\ Graduate School of Engineering, Osaka University, 2-1Yamada-oka Suita, Osaka \\ 565-0871, Japan, \\ Tel: +81-6-6879-4485, Fax: +81-6-6879-4582, \\ e-mail: murakawa@photonics.mls.eng.osaka-u.ac.jp
}

\begin{abstract}
We demonstrate a near 800 -nm range all-optical limiter to stabilize pulse-bypulse peak power fluctuations of near $800-\mathrm{nm}$ optical pulses. The all-optical limiter proposed here relies on intensity-dependent spectral pattern change by self-phase modulation (SPM) under zero-dispersion condition by using a photonic crystal fiber $(\mathrm{PCF})$. The experimental result shows the successful output power stabilization less than $0.36 \mathrm{~dB}$ against the $2.0 \mathrm{~dB}$ input power fluctuation.
\end{abstract}

Keywords: nonlinear optics, optical limiter, optical stabilizer, self-phase modulation, photonic crystal fiber

\section{Introduction}

Optical-pulse-induced ultrafast nonlinear optical effects have been widely used for various photonic applications in academic and industrial fields [1, $2,3,4,5,6,7,8]$. In the near 800-nm band, ultrafast nonlinear optical effects have often been used for optical fabrication, bio-imaging, and so on with free-space optical systems $[5,6,7,8]$, because a number of transparent materials including biological samples have a very low absorption in the near $800-\mathrm{nm}$ band [6, 7]. Since nonlinear optical effects are very sensitive to the pulse peak power, a very stable laser sources are strongly required to avoid unexpected damages due to pulse peak power fluctuations. To generate optical pulses for the free-space optical systems using nonlinear optical effects, solid-state lasers such as 800-nm Ti:sapphire lasers and 1064-nm Nd:YAG lasers have been well-developed [8]. To stabilize power fluctuations in laser 
sources, several techniques have been reported $[9,10,11,12,13]$. In the near 800-nm band, photo-detector feed-back systems have been generally adopted to stabilize power fluctuations of laser systems and such feed-back systems focus on relatively slow fluctuations (i.e., slower fluctuations than the response speed limit that can be controlled with state-of-the-art electronics). In addition, since they monitor only pulse average power fluctuations, they cannot completely avoid unexpected damages due to pulse peak power fluctuations. In the communication band, several techniques for pulse-by-pulse all-optical limiting have been proposed and demonstrated based on pulse-peak-powerdependent spectral pattern change by self-phase modulation (SPM) in a high nonlinear fiber (HNLF) $[9,11,13]$. Since SPM can be induced in the near $800-\mathrm{nm}$ band, they would be expected to effectively work on the near 800-nm band too.

In this paper, we investigate a near $800-\mathrm{nm}$ range all-optical limiter based on SPM for pulse-by-pulse power stabilization. We introduced a photonic crystal fiber (PCF) as a substitute of a HNLF in a communication band so that a SPM-based optical limiter could effectively work on the near 800$\mathrm{nm}$ band too. We used a coupling lens for coupling a collimated spatial beam from free space to a PCF. Since the fiber coupling with lenses can cause the unexpected performance of the SPM-based all-optical limiter with a PCF, we carefully examined the behavior of SPM-based spectral change. This examination is important for not only the all-optical limiter but also all the fiber-based systems when the fiber-based systems are applied to the free-space optical systems.

\section{Theoretical background}

Figure 2 shows a schematic diagram of an all-optical limiter based on selfphase modulation (SPM). As described in Fig. 2, the function is performed in the following three steps: (i) dispersion adjustment by a dispersion controller, (ii) generation of a SPM-based power-dependent spectral pattern change in a HNLF, and (iii) filtering of power-stationary spectral components by an optical band-pass filter (OBPF).

In the 1st step, a dispersive amplitude $U$ at relative time $T$ of an optical pulse after a dispersion controller is expressed as [15],

$$
U\left(T ; \beta_{2}\right)=\frac{T_{0}}{\left(T_{0}^{2}-i \beta_{2}\right)^{1 / 2}} \exp \left(-\frac{T^{2}}{2\left(T_{0}^{2}-i \beta_{2}\right)}\right),
$$




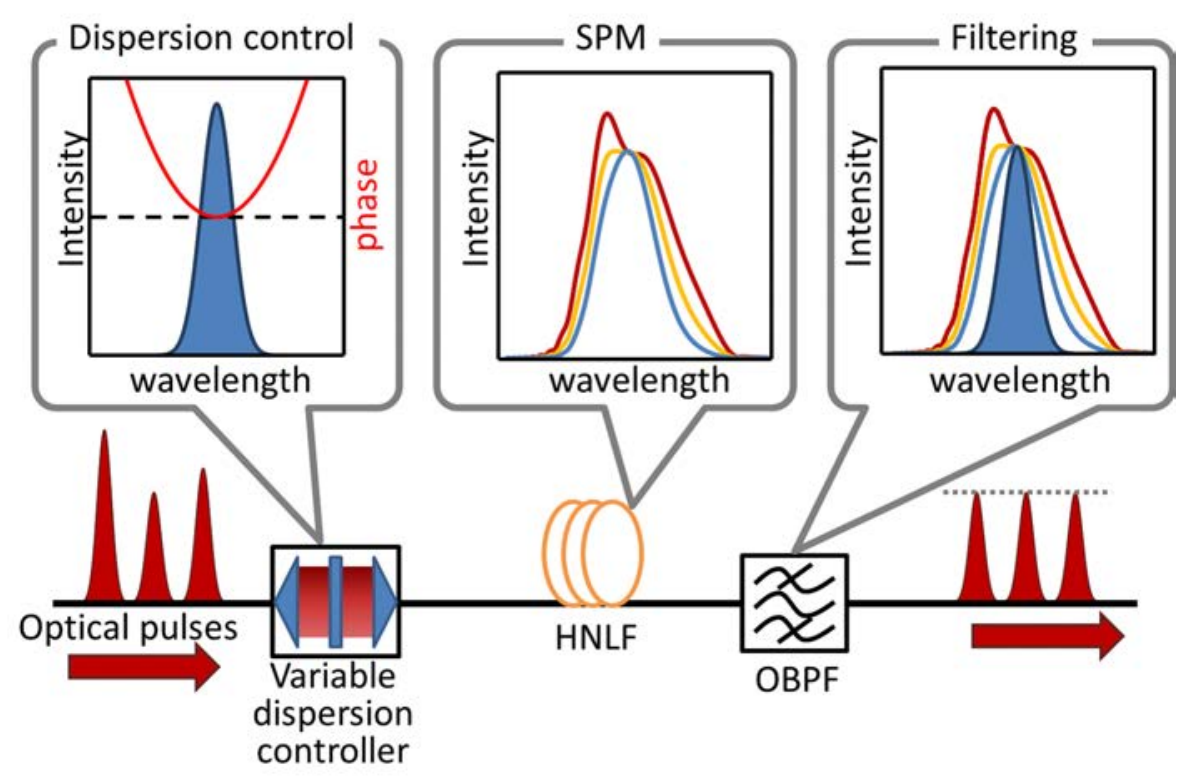

Figure 1: All-optical limiter based on SPM.

where $T_{0}$ is the half-width of the optical pulse at $1 / e$-intensity, and $\beta_{2}$ is the group delay dispersion (GDD) given by a dispersion controller. In the 2 nd step, the dispersive optical pulse is fed to a HNLF to generate a SPM-based power-dependent spectral pattern change. During propagation in a HNLF, an optical pulse experiences a certain amount of SPM-based phase shift $\phi$, which is expressed as

$$
\phi\left(z, T ; \beta_{2}\right)=\left|U\left(0, T ; \beta_{2}\right)\right|^{2}\left(\frac{L_{\mathrm{eff}}}{L_{\mathrm{NL}}}\right),
$$

where $\mathrm{z}$ is the propagation distance, $L_{\mathrm{eff}}$ is the effective propagation distance related to a fiber loss $\alpha$ by

$$
L_{\mathrm{eff}}=\frac{1-\exp (-\alpha z)}{\alpha},
$$

and $L_{\mathrm{NL}}$ is the nonlinear distance related to both the nonlinear parameter $\gamma$ and optical pulse peak power $P_{0}$ by

$$
L_{\mathrm{NL}}=\left(\gamma P_{0}\right)^{-1}
$$




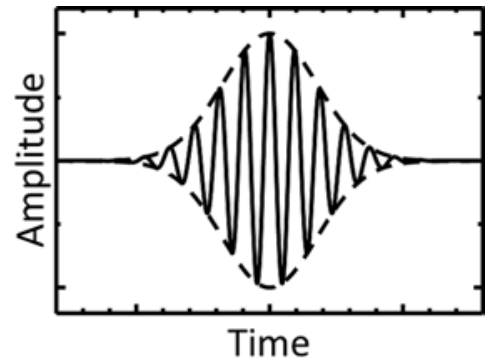

(a)

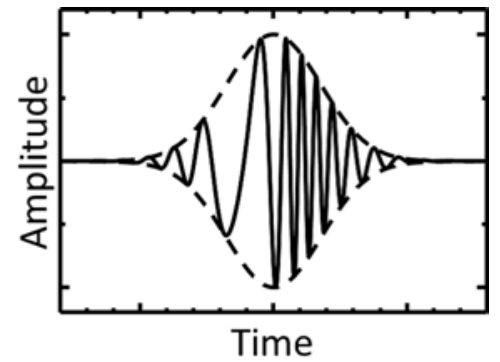

(b)

Figure 2: Temporal phase in an optical pulse (a) before and (b) after SPM-based phase shift.

From equation (2), SPM-based phase shift $\phi$ is proportional to $\left|U\left(0, T ; \beta_{2}\right)\right|^{2}$ and it is a function of $\beta_{2}$ as well as $T$. Figure 2 shows an apparatus of a SPM-based phase shift which results in changing a spectrum of a transform limited optical pulse. The instantaneous frequency component of an optical pulse changes according to its instantaneous power because it is given by a differential of a SPM-based phase shift $\phi$ as described by equation

$$
\delta \omega\left(T ; \beta_{2}\right)=-\frac{\partial \phi}{\partial T}=-\frac{\partial\left(\left|U\left(0, T ; \beta_{2}\right)\right|^{2}\right)}{\partial T} \frac{L_{\mathrm{eff}}}{L_{\mathrm{NL}}} .
$$

From equation (5), a SPM-based power-dependent spectral pattern change of an optical pulse can be controlled by adjusting the initial value of GDD $\beta_{2}$ of an optical pulse.

In the 3rd step, the optical pulse after the spectral change is fed to an OBPF to extract the almost stationary power components out of the optical pulse spectral components. Since the output pulse after the OBPF could keep a certain stationary power with no reference to an input power change, the function of an all-optical limiter is completed. In general, an optical pulse in the near 800-nm band cannot effectively induce SPM in a conventional HNLF in the communication band because the zero-dispersion wavelength of such a HNLF is much longer one than the near $800-\mathrm{nm}$ band and GDD is too large to induce SPM. To solve this issue, we examine a PCF in the near $800-\mathrm{nm}$ band to see how it can serve as a substitute of a HNLF in a communication band. A PCF is a kind of a silica fiber with an array of micro-scopic air holes along its distance, and a specific characteristics of the structure is expected to provide zero-dispersion condition in the near $800-\mathrm{nm}$ band. However, 


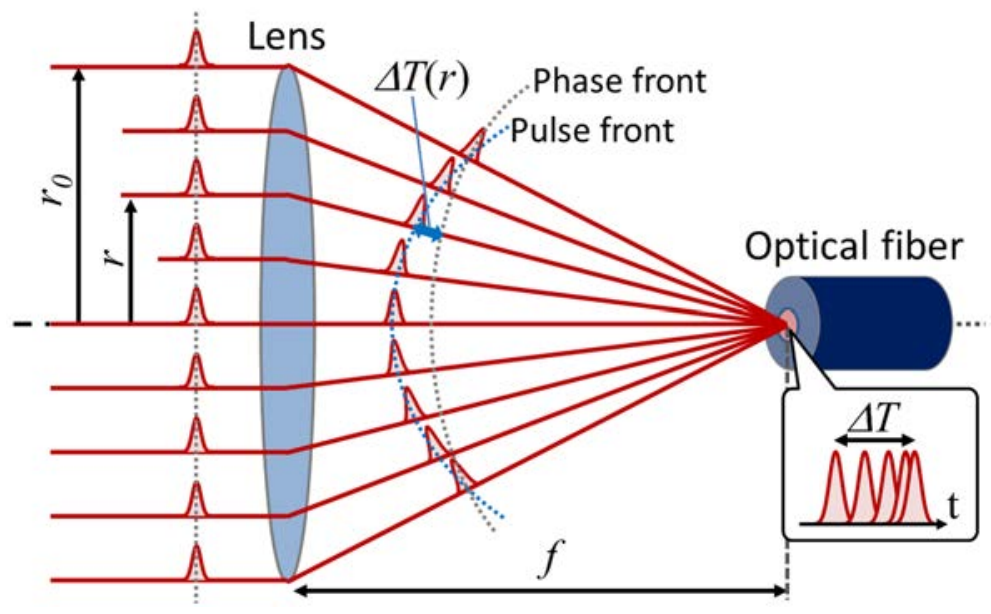

Figure 3: Coupling by a biconvex singlet lens.

the beam coupling with a coupling lens is essential for the usage of a PCF because a standard fiber connector generally does not well match to a bare PCF and an optical pulse in the near $800-n m$ band is mostly provided as a collimated spatial beam radiated from a solid state laser too. Figure 3 shows the apparatus of the beam coupling and phase and pulse fronts of an optical pulse does not match after a coupling lens[16]. Since a coupling lens could lead to such a distortion of an optical pulse with spatially structured spatiotemporal dispersion, we need to carefully examine the behavior of SPM-based spectral change prior to the usage of PCF for optical limiting.

\section{Simulation}

We investigated the behavior of SPM-based spectral change in a PCF depending on an input pulse power. Figure 4 shows the power spectrum of the input optical pulse used for simulation which is same as that in an experiment.

Here, we assumed its initial phase to be 0. For comparison, we used a HNLF in a communication band and a PCF in a near $800-\mathrm{nm}$ band. The parameters of the HNLF used for simulation at $1550 \mathrm{~nm}$ were following: fiber length $L=20 \mathrm{~m}$, dispersion parameter $D=-0.04 \mathrm{ps} / \mathrm{nm} / \mathrm{km}$ (or -364.84 $\mathrm{ps} / \mathrm{nm} / \mathrm{km}$ at $790 \mathrm{~nm}$ ), dispersion slope $S l=0.48 \mathrm{ps} / \mathrm{nm}^{2} / \mathrm{km}$, nonlinear parameter $\gamma=14.4 / \mathrm{W} / \mathrm{km}$, loss $\alpha=0.19 \mathrm{~dB} / \mathrm{km}$. The parameters of the PCF used for simulation at $790 \mathrm{~nm}$ were following: fiber length $L=$ 


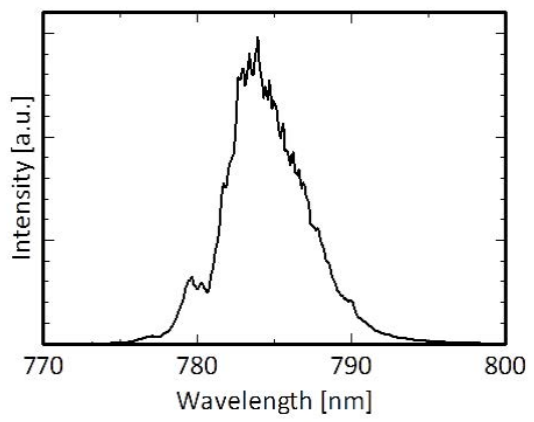

Figure 4: Spectrum of input optical pulse from the actual fiber laser.

$3 \mathrm{~m}$, dispersion parameter $D=0 \mathrm{ps} / \mathrm{nm} / \mathrm{km}$, dispersion slope $S l=0.64$ $\mathrm{ps} / \mathrm{nm}^{2} / \mathrm{km}$, nonlinear parameter $\gamma=75 / \mathrm{W} / \mathrm{km}$, loss $\alpha=22.193 \mathrm{~dB} / \mathrm{km}$. Figure 5(a) and (b) are spectral changes for a HNLF and a PCF, respectively and effective SPM-based spectral changes were generated only for the PCF. While we can directly measure only a pulse average power, actual SPM-based spectral change certainly depend on a pulse peak power. Henceforward, representation of average power is used for explanation instead of that of peak power because a pulse peak power can be easily calculated from a pulse average power. From the results in Fig. 5, a PCF in the near 800-nm band can serve as a substitute of a HNLF for the all-optical limiter in near $800 \mathrm{~nm}$. When $\beta_{2}$ is set at $0.03-\mathrm{ps}^{2}$, spectral components around $782 \mathrm{~nm}$ remained at a certain stationary power with no reference to an input power change, as shown in Fig. 5(b).

To confirm the input-output characteristics of an all-optical limiter with a PCF, the optical pulse after SPM-based spectral change was filtered by a gaussian $\mathrm{OBPF}$ with a $3-\mathrm{dB}$ bandwidth of $3 \mathrm{~nm}$ and a center wavelength of $782 \mathrm{~nm}$. Figure 6 shows the simulation results of the input-output characteristics of an all-optical limiter. A typical optical limiting characteristic successfully can be observed when $\beta_{2}$ of the input pulse is set at $0.03 \mathrm{ps}^{2}$.

Next, we investigated influences depending on the presence or absence of a coupling lens. The simulation condition is same as those in the above simulation except the presence of a coupling lens. Here, we assume a biconvex singlet lens as a typical coupling lens for a beam coupling simulation. The entrance pupil diameter, the focal length and the glass material of a coupling lens are assumed to be $11.6 \mathrm{~mm}, 4.5 \mathrm{~mm}$, and BK7, respectively. A spatially 


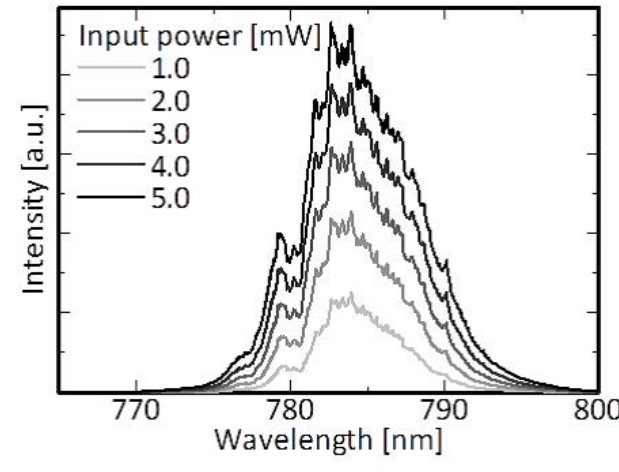

(a)

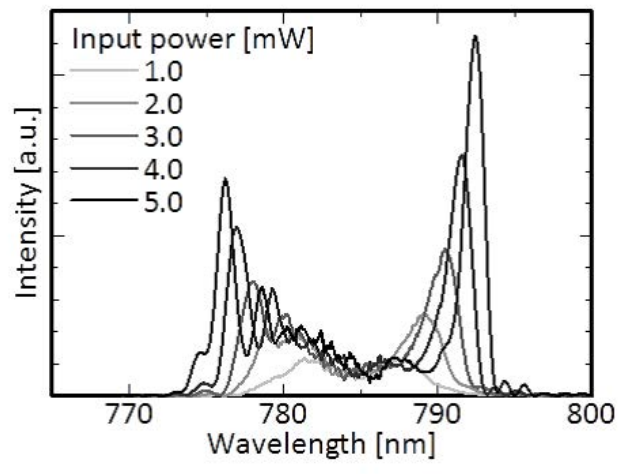

(b)

Figure 5: Spectral pattern change of (a) the 782-nm transform-limited optical pulse after a conventional HNLF with zero dispersion wavelength at $1550 \mathrm{~nm}$, and (b) the 782-nm $0.03-\mathrm{ps}^{2}$ dispersive optical pulse after a PCF with zero dispersion wavelength at $790 \mathrm{~nm}$.

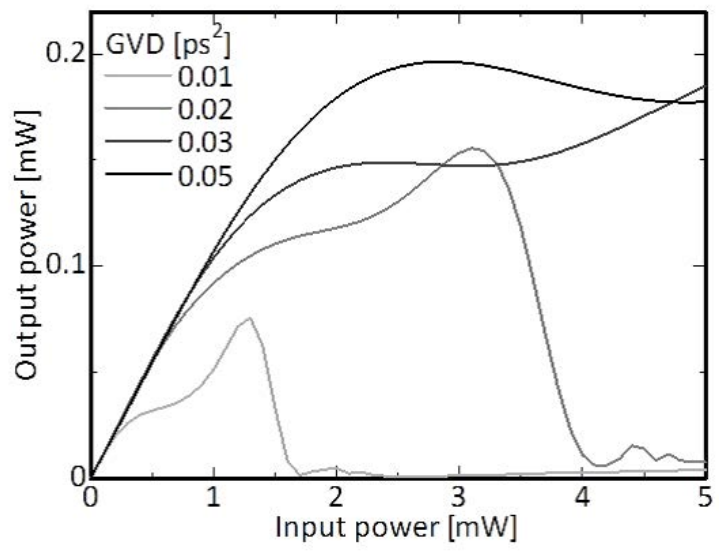

Figure 6: Simulation results of the input-output characteristics of the all-optical limiter. 


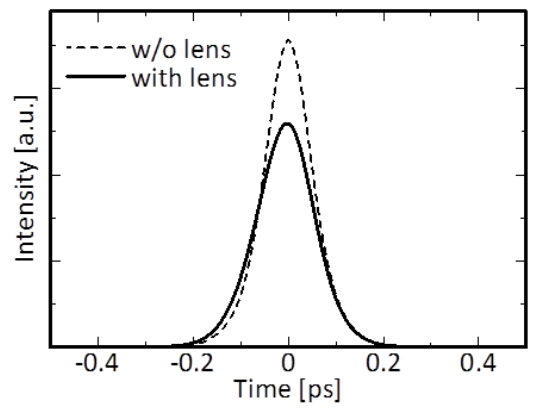

Figure 7: Temporal waveform of an optical pulse with and without presence of a biconvex singlet lens.

structured group delay $\Delta T$ due to a biconvex singlet lens is given by,

$$
\Delta T=\frac{r_{0}^{2}-r^{2}}{2 c f(n-1)}\left(-\lambda \frac{\mathrm{d} n}{\mathrm{~d} \lambda}\right)
$$

where $r_{0}, r, c, f, n, \lambda, \mathrm{d} n / \mathrm{d} \lambda$ are, the radius of the lens, the input radius of an arbitrary ray entering the lens, the velocity of light, the focal length, the refractive index, and the wavelength of the input optical pulse, respectively [16]. Here, we neglected the influence of GDD because it is estimated to be less than a few attoseconds. Figure 7 shows the temporal waveforms of an optical pulse with and without presence of a biconvex singlet lens. The peak power decreases and the pulse width broadens after passing through a biconvex singlet lens.

We investigated the behavior of SPM-based spectral change in a PCF by considering the influence of the spatially structured group delay $\Delta T$. Figure 8 shows the SPM-based spectral change depending on an input pulse average power. We can certainly see a small difference depending on the presence or absence of a coupling lens as compared with Fig. 5 (b). However, the influences are not large and the stationary average power is still observed around $782 \mathrm{~nm}$.

To confirm the input-output characteristics of an all-optical limiter with a PCF and a coupling lens, the optical pulse after SPM-based spectral change was filtered by a gaussian OBPF with a $3-\mathrm{dB}$ bandwidth of $3 \mathrm{~nm}$ and a center wavelength of $782 \mathrm{~nm}$. Figure 9 shows the simulation results of the inputoutput characteristics of an all-optical limiter with and without presence of a biconvex singlet lens when $\beta_{2}$ of the input pulse is set at $0.03 \mathrm{ps}^{2}$. From 




Figure 8: Spectral pattern change of the $0.03-\mathrm{ps}^{2}$ dispersive optical pulse in the simulation with a biconvex singlet lens.

these results, we can see that the influence of a lens is not large and sufficient input-output characteristic for the all-optical limiter is obtained under our current experimental condition.

\section{Experiment}

We performed an experiment using a 3-m PCF which has the same parameters as used in the simulation to demonstrate a near $800-\mathrm{nm}$ range all-optical limiter. Figure 10 shows the experimental setup. We used an optical pulse generated by a fiber-laser (IMRA America: femtolite 780) as a light source with a repetition rate of $50 \mathrm{MHz}$, a pulse width of $0.14 \mathrm{ps}$ and a center wavelength of $782 \mathrm{~nm}$. The optical pulses passed through a pulse shaper to be given an appropriate GDD. The power of the appropriately dispersive optical pulse was regulated by a variable optical attenuator (VOA). Subsequently, the appropriately dispersive optical pulses are coupled into the PCF using an objective lens, and then propagated in the PCF. The optical pulses from the PCF was collimated by a lens and passed through an OBPF to filter the stationary average power components. The spectra of the filtered optical pulses are measured by a spectrometer to observe the input-output characteristics of the all-optical limiter.

Figure 11 shows the spectral pattern change of the dispersive optical pulses by propagation in the PCF. Here, we gave the GDD of $0.00156 \mathrm{ps}^{2}$ to the optical pulses based on results of preliminary experiments.

Figure 12 shows the experimental result of the input-output characteristics of the all-optical limiter. The output power was calculated from the 


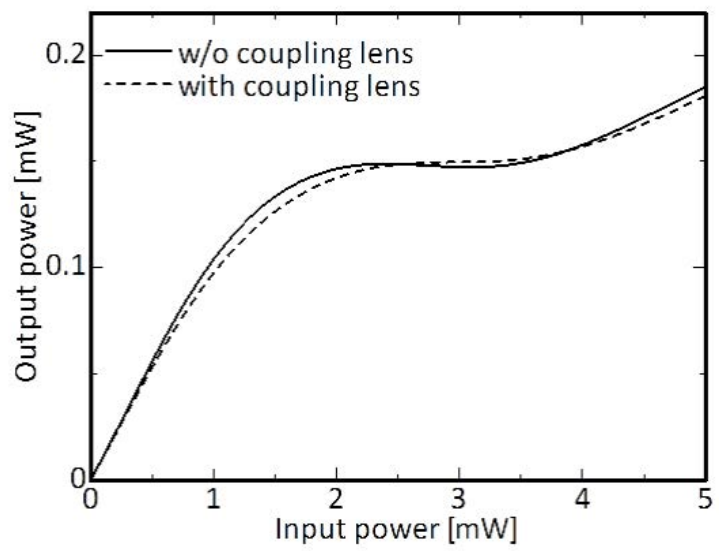

Figure 9: Comparison of the input-output characteristics of the all-optical limiter at 0.03 $\mathrm{ps}^{2}$ between with and without a biconvex singlet lens.

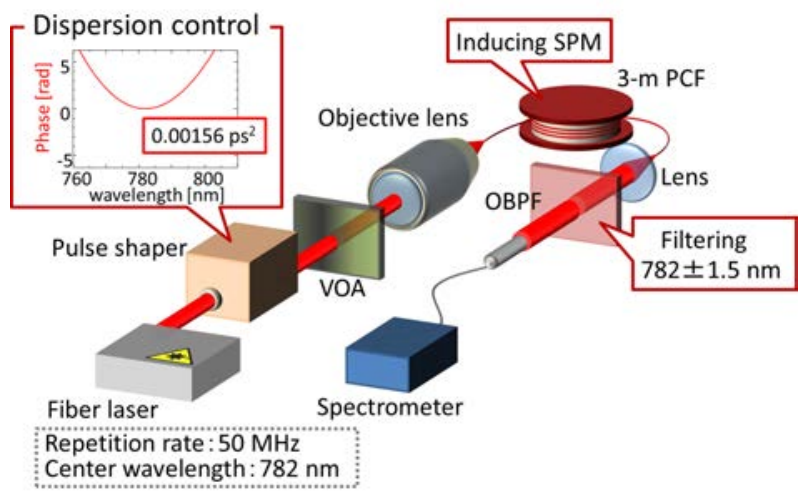

Figure 10: Experimental setup. 


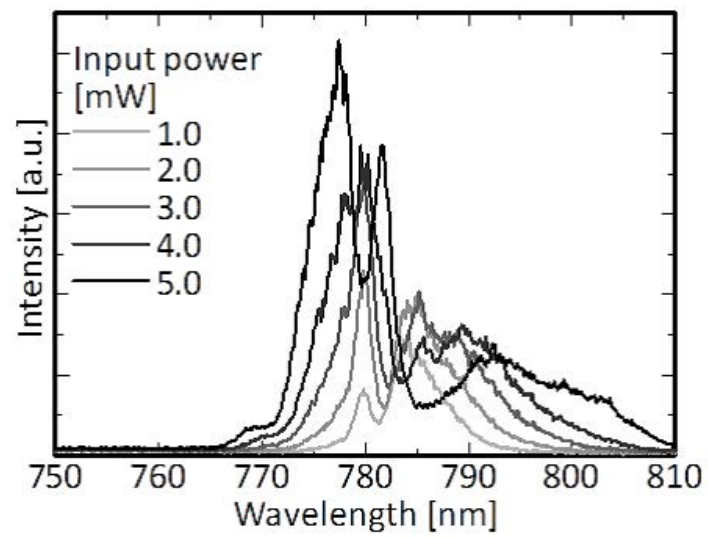

Figure 11: Spectral pattern change of the dispersive optical pulses in the experiment.

integration of the measured spectrum. Figure 12 also indicates that the power fluctuation of the output optical pulse is less than $0.36 \mathrm{~dB}$ for the input average power fluctuation from $2.4 \mathrm{~mW}$ to $3.8 \mathrm{~mW}(2.0-\mathrm{dB}$ input average power range). From these results, we confirmed the basic function of the near 800-nm range all-optical limiter based on SPM. The difference of the optimized GDD between the simulation and the experiment was due to the difference of the initial phase of the input optical pulse.

\section{Conclusions}

We investigated a near 800-nm range all-optical limiter based on SPM by introducing a PCF as a substitute of a HNLF in a communication band and carefully examined the influence of a spatially structured spatio-temporal pulse distortion at the beam coupling. The experimental result shows the successful output power stabilization less than $0.36 \mathrm{~dB}$ against the $2.0 \mathrm{~dB}$ input power fluctuation. We conclude that pulse-by-pulse all-optical limiting can be achieved through a PCF in near $800-\mathrm{nm}$ band and there appears to be a great potential for applications in the near future for various photonic applications. 


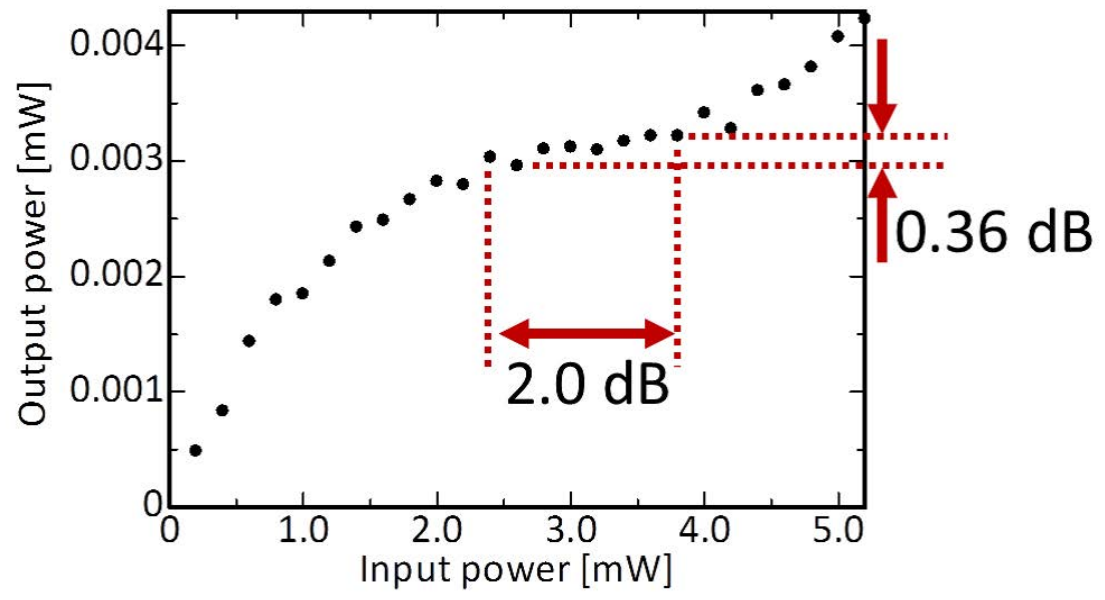

Figure 12: Experimental result of the input-output characteristics of the all-optical limiter in the near 800-nm band.

\section{Acknowledgement}

This research is partially supported by the Center of Innovation Program from Japan Science and Technology Agency, JST.

\section{Reference}

[1] B. J. Eggleton, B. Luther-Davies, and K. Richardson, Nat. Photon. 5 (2011), 141-148.

[2] E. Palushani, H. C. Hansen Mulvad, D. Kong, P. Guan, M. Galili, and L.K. Oxenløwe, Opt. Express, 22(2014) 136-144.

[3] T. Konishi, K. Tanimura, K. Asano, Y. Oshita, and Y. Ichioka, JOSA. B 19 (2002) 2817-2823.

[4] M. A. Foster, R. Salem, D. F. Geraghty, A. C. Turner-Foster, M. Lipson, and A. L. Gaeta, Nature 456 (2008) 81-84.

[5] R. R. Gattass and E. Mazur, Nat. Photonics 2 (2008) 219-225.

[6] W. R. Zipfel, R. M. Williams, W. W. Webb, Nat. Biotechnol. 21 (2003)1369-1377. 
[7] J. G. Fujimoto and D. L. Farkas, Biomedical Optical Imaging, Oxford University Press, New York, 2009.

[8] K. Sugioka, M. Meunier, and A. Pique, Laser Precision Microfabrication, Springer-Verlag, Berlin Heidelberg, 2010.

[9] T. Ohara, H. Takara, S. Kawanishi, T. Yamada, and M. M. Fejer, IEEE Photonics Technol. Lett. 16 (2004) 2311-2313.

[10] G. Contestabile, M. Presi, R. Proietti, N. Calabretta and E. Ciaramella, Opt. Express 15 (2007) 9849-9858.

[11] H. Goto, T. Konishi and K. Itoh, Jpn. J. Appl. Phys. 10 (2008) 88348837.

[12] Q. Zheng, G.S. He, and P.N. Prasad, Chem. Phys. Lett. 475 (2009) 250-255.

[13] K. Kawanishi, T. Konishi and K. Itoh, IEEE Photonics Technology Letters 24 (2012) 119-121.

[14] P.St.J. Russell, Science 299 (2003) 358-362.

[15] G. P. Agrawal, Nonlinear Fiber Optics 4th edition, Academic Press, San Diego, CA, 2006.

[16] Z.Bor, J. Mod. Opt. 35 (1988) 1907-1918. 